\title{
Comparison of artificial neural network and logistic regression models for prediction of mortality in head trauma based on initial clinical data

\author{
Behzad Eftekhar*1, Kazem Mohammad², Hassan Eftekhar Ardebili³, \\ Mohammad Ghodsi ${ }^{1}$ and Ebrahim Ketabchi ${ }^{1}$
}

Address: ${ }^{1}$ Department of Neurosurgery, Sina Hospital, Tehran University, Tehran, Iran, ${ }^{2}$ Department of Biostatistics and Epidemiology, Faculty of Public Health, Tehran University, Tehran, Iran and ${ }^{3}$ Department of Public Health, Faculty of Public Health, Tehran University, Tehran, Iran

Email: Behzad Eftekhar* - eftekhar@sina.tums.ac.ir; Kazem Mohammad - mohammad@sina.tums.ac.ir;

Hassan Eftekhar Ardebili - hasaneftekhar@yahoo.com; Mohammad Ghodsi - ghodsism@sina.tums.ac.ir; Ebrahim Ketabchi - ebrahimketabchi@yahoo.com

* Corresponding author

Published: 15 February 2005

BMC Medical Informatics and Decision Making 2005, 5:3 doi:10.1 186/1472-6947-5-3

This article is available from: http://www.biomedcentral.com/1472-6947/5/3

(c) 2005 Eftekhar et al; licensee BioMed Central Ltd.

This is an Open Access article distributed under the terms of the Creative Commons Attribution License (http://creativecommons.org/licenses/by/2.0), which permits unrestricted use, distribution, and reproduction in any medium, provided the original work is properly cited.
Received: 24 August 2004

Accepted: 15 February 2005

\begin{abstract}
Background: In recent years, outcome prediction models using artificial neural network and multivariable logistic regression analysis have been developed in many areas of health care research. Both these methods have advantages and disadvantages. In this study we have compared the performance of artificial neural network and multivariable logistic regression models, in prediction of outcomes in head trauma and studied the reproducibility of the findings.
\end{abstract}

Methods: 1000 Logistic regression and ANN models based on initial clinical data related to the GCS, tracheal intubation status, age, systolic blood pressure, respiratory rate, pulse rate, injury severity score and the outcome of I27I mainly head injured patients were compared in this study. For each of one thousand pairs of ANN and logistic models, the area under the receiver operating characteristic (ROC) curves, Hosmer-Lemeshow $(\mathrm{HL})$ statistics and accuracy rate were calculated and compared using paired T-tests.

Results: ANN significantly outperformed logistic models in both fields of discrimination and calibration but under performed in accuracy. In $77.8 \%$ of cases the area under the ROC curves and in $56.4 \%$ of cases the HL statistics for the neural network model were superior to that for the logistic model. In $68 \%$ of cases the accuracy of the logistic model was superior to the neural network model.

Conclusions: ANN significantly outperformed the logistic models in both fields of discrimination and calibration but lagged behind in accuracy. This study clearly showed that any single comparison between these two models might not reliably represent the true end results. External validation of the designed models, using larger databases with different rates of outcomes is necessary to get an accurate measure of performance outside the development population. 


\section{Background}

In recent years, outcome prediction studies have become the avante garde in many areas of health care research, especially in critical care and trauma. However acceptable models for outcome prediction have been difficult to develop [1]. According to Wyatt and Altman, to be useful, a predictive model must be simple to calculate, have an apparent structure and be tested in independent data sets with evidence of generality [2]. While this is a high standard, availability and popularity of portable computers, deprioritize the need for simplicity of the model and having an apparent structure.

Artificial neural networks (ANNs) are mathematical constructs modeled on interconnection of nodes (neurons) giving a loose association with the animal nervous system. [3]

ANNs employ nonlinear mathematical models to mimic the human brain's own problem-solving process. Just as humans apply knowledge gained from past experience to new problems or situations, a neural network takes previously solved examples to build a system of "neurons" that makes new decisions, classifications, and forecasts. [4]

ANNs are complex and flexible nonlinear systems with properties not found in other modeling systems. These properties include robust performance in dealing with noisy or incomplete input patterns, high fault tolerance, and the ability to generalize from the input data [5]. Neural networks excel at applications where pattern recognition is important, and precise computational answers are not required, such as forecasting weather, stock predicting, or speech recognition [6]. Reports in medical literature suggest that ANN models are applicable in diagnosing diseases such as myocardial infarction $[7,8]$ pulmonary emboli detection [9], gastrointestinal hemorrhage [10], waveform analysis of EKGs [11], EEGs [12,13], and radiographic images [14]. ANNs have also been successfully applied in clinical outcome prediction of trauma mortality $[1,15]$, surgical decision making on traumatic brain injury patients $[16]$, recovery from surgery $[17,18]$, outcome in pediatric meningococcal disease [19] and transplantation outcome [20].

Lang EW et al have compared ANN with Logistic Regression in prediction of outcome after severe head injury and concluded that the differences in the results obtained with the two models were negligible [21].

Almost all of the published articles indicate that the performance of ANN models and logistic regression models have been compared only once in a dataset and the essential issue of internal validity (reproducibility) of the models has not been addressed.
The objective of this study was to compare the performance of ANN and multivariate logistic regression models for prediction of mortality in head trauma based on initial clinical data and whether these models are reproducible. We used different variables even if they were interdependent.

\section{Methods \\ Study population}

Among 8452 trauma patients' records admitted to the emergency departments of six major university hospitals in Tehran from 23 August 1999 to 22 September 2000, the records of 1271 patients whose main trauma was head injury, were selected for this study. The selection of head trauma as the main trauma was based on the definition of principal diagnosis in the Uniform Hospital Discharge Data Set (UHDDS). It defines the principal diagnosis as "that condition established after study to be chiefly responsible for occasioning the admission of the patient to the hospital for care". For making determination of the main trauma more practical in the case of ambiguity, hospitalization in the neurosurgical ward was used as an additional guideline. The database was based upon the trauma data registry program began in 1996 in Trauma Research Center, Sina Hospital, a hospital affiliated with the Tehran University of Medical Sciences [22,23]. The study population for this study was comprised of all trauma victims who had been admitted in one of the hospitals for more than 24 hours during the data-gathering period. For dead patients, this time limitation was disregarded, that is, records of all dead patients who were referred to these hospitals were included in the study. We have excluded those transferred to other hospitals or with related missing values. Structured, closed-question data checklists were used for the data gathering process. Three major categories of injury-related information were collected, that is, demographic data, pre-hospital data (if they were available) and in-hospital data. Hospital related data included: vital signs, Glasgow Coma Scale (GCS), Abbreviated Injury Scale (AIS-90 [24]), clinical findings in accordance with the International Classification of Diseases $10^{\text {th }}$ revision (ICD-10) as well as the outcome of the patients. Data collection was conducted by a group of trained physicians who had completed special training courses to become familiar with the process of extracting Abbreviated Injury Score (AIS-90) codes and filling out the relevant questionnaires. For quality control (QC) purposes each hospital had a physician, who was responsible for overseeing the data gathering process. In the intubated patients GCS were calculated according to the other portions of the scale by this physician. Finally, a medical practitioner examined all the checklists in order to evaluate and amend them if deemed necessary based on prearranged and fixed protocols. 


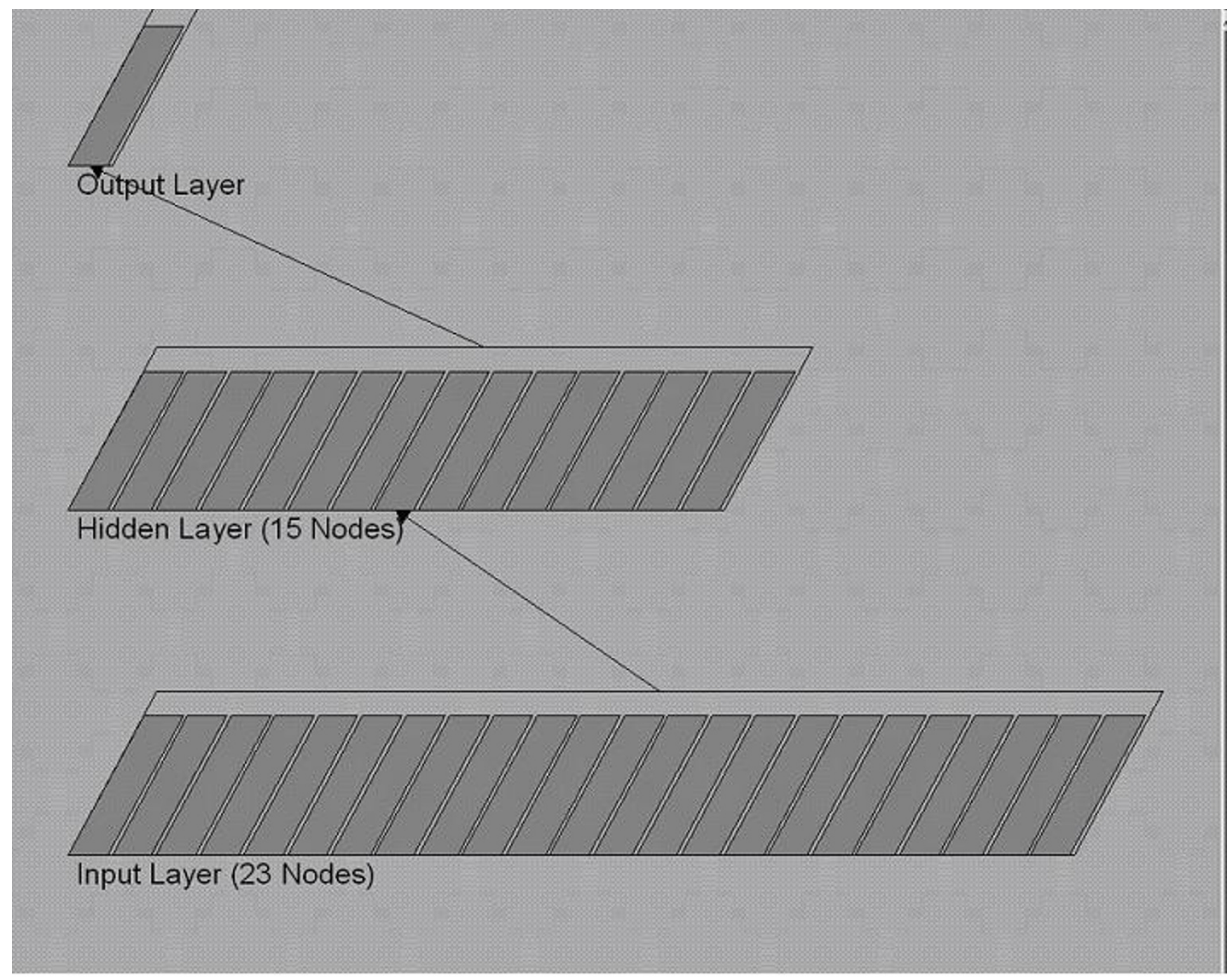

\section{Figure I}

Diagrammatic representation of Artifical Neural Network ANN) structure with 23 input nodes in the input layer, 15 nodes in the hidden layer and one node in the output layer.

Since we were trying to build and compare models for prediction of outcome mainly based on the initial clinical data, only data related to the GCS, tracheal intubation status, age, systolic blood pressure (SBP), respiratory rate(RR), pulse rate(PR), injury severity score (ISS)(upon admission) and outcome were used in our study. In order to prepare the data for the Neural Network software and to enhance the reliability of the data, three variables of systolic blood pressure, respiratory rate and pulse rate were transformed to dichotomous $(1,0)$ variables. Low systolic blood pressure was defined according to the following cutoff points: up to 5 years of age, less than 80 $\mathrm{mmHg}$; and 5 years of age or older, less than $90 \mathrm{mmHg}$.
Respiratory rate of 35 per min and pulse rate of 90 per min were selected as limits for definition of tachypnea and tachycardia. Other variables including GCS, age, systolic blood pressure (SBP) and injury severity score (ISS) variables were also converted from decimal (Base 10) to binary (Base 2). This conversion was carried out in order to render the input data suitable for processing by our ANN software with its default settings. The data and the data format were similar for both ANN and logistic regression models. 


\section{Development of logistic regression models}

The dataset was divided randomly into two sets, one set of 839 cases ( $66 \%$ of the whole dataset) for training and 432 cases for testing the model. A model was built using a training set with logistic regression. GCS, tracheal intubation status(dichotomous), age, SBP(dichotomous), $\mathrm{RR}$ (dichotomous), PR(dichotomous) and ISS were the independent variables and the outcome (death/survival) was the dependent variable. The logistic regression analyses were performed using Intercooled STATA for windows, Version 6 (STATA Corp., College Station, TX) "logistic" default options.

The built logistic model was tested using the testing dataset (432 cases). These steps (randomized division of dataset and regression analysis considering the same variables) were repeated 1000 times. This resulted in 1000 pairs of training and testing datasets $(2 / 3$ and $1 / 3$ of the original dataset, respectively) which were saved for further processing by the neural networking.

\section{Development of ANN models}

The ANN used in this study was a standard feed-forward, back-propagation neural network with three layers: an input layer, a hidden layer and an output layer. The input layer consisted of 23 input neurons, the hidden layer consisted of fifteen hidden neurons, and the output layer consisted of one output neuron (Fig. 1). The learning rate and momentum for network training were set respectively to 0.25 and 0.9 and the models were run until a minimum average squared error $<0.063$ was obtained. The number of the network layers, hidden neurons and the stopping criteria were determined through trial-and-errors process because no commonly accepted theory exists for predetermining the optimal number of neurons in the hidden layer [25].

The training and testing datasets were the same as those were used with regression models, thus there was an ANN and a logistic model for each training and testing dataset.

PDP++ version 3.1 was used for the artificial neural network analysis as it has a powerful built-in scripting language and is freely available. This software can be downloaded from ftp://cnbc.cmu.edu/pub/pdp++/.

\section{Comparison of model performance}

Discrimination and calibration (goodness of fit) were both measured. Discrimination refers to the ability of a model to distinguish those who die from those who survive. A perfectly discriminating model would assign a higher probability of death to all cases that died than to any case that survived. The discriminatory power of the models was analyzed using the area under the receiver operating characteristic curves (ROC-A(z)). ROC curves
Table I: General Characteristics of the Dataset

\begin{tabular}{ll}
\hline GCS & $13.5 \pm 3$ \\
Sex & $76 \%$ Male \\
ISS & $9.5 \pm 14$ \\
SBP & $117 \pm 23$ \\
RR & $19 \pm 7$ \\
PR & $86 \pm 17$ \\
Intubated & $2 \%$ \\
Age & $28.5 \pm 19$ \\
Mortality & $7.5 \%$
\end{tabular}

GCS = Glasgow Coma Scale; ISS = Injury Severity Score; SBP = Systolic Blood Pressure; RR = Respiratory Rate; PR = Pulse Rate

were constructed by plotting true positives (patients who died and whom the model predicted as dying [i.e. sensitivity]) versus the false positive fraction (fraction of the patients who lived and were incorrectly classified as dying [i.e. (1 - specificity)]).

A ROC-A $(z)$ value of one corresponds to a test that perfectly separates two populations, whereas a ROC-A(z) value of 0.5 corresponds to a perfectly useless test that performs no better than chance.

The relative calibration of the models, that is, how accurately the models predicted over the entire range of severity, was compared using Hosmer-Lemeshow (HL) statistics. The HL statistics is a single summary measure of the calibration and is based on comparing the observed and estimated mortality for patients grouped by estimated mortality. The resulting statistic follows a chi-squared distribution, with degrees of freedom equal to two less than the number of groups ( 10 in this study). The smaller the HL statistics, the better the fit, with a perfectly calibrated model having a value of zero.

A probability cut point of 0.5 was used to classify observations as events or nonevents. The overall accuracy ([true positive+true negative]/total) of the final model was determined by comparing the predicted values with the actual events.

For each of one thousand pairs of ANN and logistic models (trained and tested on the same datasets), these indices (area under the ROC curves, HL statistics and accuracy rate) were calculated and compared using paired T-tests ( $P$ $<0.05)$.

All the statistical analyses were performed using Intercooled STATA for windows, version 6 STATA Corp., College Station, TX) and its downloadable add-on ado files. 
Table 2: Results of Comparing 1000 pairs of Artificial Neural Network (ANN) and Logistic Regression (LR) models

\begin{tabular}{llll}
\hline & LR(95\% Confidence Interval) & ANN(95\% Confidence Interval) & $P<$ \\
\hline Area under ROC curve & $.9538(.9527-.9549)$ & $.9646(.9627-.9665)$ & 0.0000 \\
H-L Statistics & $53.13(46.04-60.23)$ & $41.51(32.92-50.11)$ & 0.015 \\
Accuracy Rate & $96.37(96.32-96.42)$ & $95.09(94.93-95.24)$ & 0.0000 \\
\hline
\end{tabular}

Some of the scripts used both for the STATA and PDP++ and the designed neural networks can be downloaded from the site of the Tehran University of Medical Sciences http://www.tums.ac.ir/download. Further details are available from the corresponding author.

\section{Results}

Table 1 shows clinical characteristics of the dataset. The mean age of the study population was $(28.5 \pm 19)$ years. $76 \%$ of our patients were male and the overall mortality rate was $7.5 \% .7 .5 \%$ of the patients had GCS $<8$.

As is seen in Table 2 ANN significantly outperformed logistic models in both senses of discrimination and calibration, although from the standpoint of accuracy (cutoff point 0.5 ), logistic models were superior to ANN models. In $77.8 \%$ of cases the area under the ROC curve and in $56.4 \%$ of cases the HL statistics for the neural network model were superior to that for the logistic model. In $68 \%$ of cases the accuracy of the logistic model was superior to the neural network model.

The confidence intervals of the all the measures show higher variation in the ANN models results.

\section{Discussion}

Prognostic evaluation of the patients without CT scan findings may have limited applicability considering the availability of CT scans in the majority of the trauma centers. Nevertheless, the idea of prognostic models based on initial clinical data at admission is still worth trying and seems to be of practical value in some situations. Although omission of CT data weakens our armamentarium significantly, patients without paraclinical and imaging data make the real trauma scenes at which medical staff should have an evaluation. Actually as neurosurgeons we have initial clinical judgments based on our growing experiences and in many situations they prove to be true. We can expect computers do similar things and help us. In fact, vague situations like what we see in primary evaluation of head trauma patients are where ANN may prove to be superior to traditional linear modeling. This is one of the reasons that although many paraclinical and imaging factors are known to be of significant predic- tive value in the outcome of the head trauma patients, this study only used clinical measures which are simply available to a physician in the emergency department.

\section{Study limitations}

Calculating ISS and AIS are not simple tasks and needs training. This can reduce the practicability of the results. The pupillary size and reactivity are one of the clinical signs with prognostic value that were not considered in the study due to defect of the main database in this regard.

As is seen the mortality rate and the percentage of patients with GCS $<8$ are the same $(7.5 \%)$. This is a coincidence, but emphasizes the need for further studies in the populations with different rates of outcome.

The mean GCS was $13.5 \pm 3$. The standard deviation is over the top score of GCS (15) and means that the GCSs were skewed towards higher levels of consciousness.

Not considering the exact time interval between head trauma and admission which is simply available for the medical staff is one of the weak points of this study.

The lower intubation rate in this population study related to the distribution of the GCS may show the lower quality of the pre-hospital care prevailing in the hospitals selected for this study. This may render the reproduction of the results using the same network (Downloadable from http://www.tums.ac.ir/download) somewhat difficult in other situations where pre-hospital care services are more advanced.

Using ISS as an independent variable underlines the role of general trauma in the models used for this study. This was unavoidable, because the original dataset had been a subset of general trauma patients.

\section{Comparison of two models}

Currently, the logistic regression and the artificial neural networks are the most widely used models in biomedicine, as measured by the number of publications indexed in Pubmed as attested by 45646 cases for the logistic regression and 8015 for the neural network. 
Logistic regression is a commonly accepted statistical tool, which can generate excellent models. Its popularity may be attributed to the interpretability of model parameters and ease of use, although it has limitations. For example, logistic regression models use linear combinations of variables and, therefore, are not adept at modeling grossly nonlinear complex interactions as has been demonstrated in biologic and complex epidemiologic systems.

Not withstanding its limitations, neural networks are appealing for a number of reasons, namely; they seem to "learn" without supervision, they can be created by workers with very little mathematical model building experience, and software for building neural networks is now readily available. Neural networks have perhaps a special appeal to the medical community because of their superficial resemblance to the human brain (a structure with which most physicians are comfortable), and seem to promise "prediction" without the difficulties associated with use of mathematics.

ANNs are rich and flexible nonlinear systems that show robust performance in dealing with noisy or incomplete data and have the ability to generalize from the input data. They may be better suited than other modeling systems to predict outcomes when the relationships between the variables are complex, multidimensional, and nonlinear as found in complex biological systems.

The difficulty in developing models using artificial neural networks is that there are no set methods for constructing the architecture of the network. The most common type of artificial neural networks is the feed-forward back propagation multiperceptron (used in this study).

Another limitation of neural network models is that standardized coefficients and odds ratios corresponding to each variable cannot be easily calculated and presented as they are in regression models. Neural network analysis generates weights, which are difficult to interpret as they are affected by the program used to generate them [26]. This lack of interpretability at the level of individual variables (predictors) is one of the most criticized features in neural network models [27].

Several early applications of neural networks in medicine reported an excellent fit of the ANN model to a given set of data. The impressive results usually were derived from over fitted models, where too many free parameters were allowed. Linear and logistic regression models have less potential for overfitting primarily because the range of functions they can model is limited.

Neural network models require sophisticated software. The complexity and unfamiliarity of ANN has been a major drawback of this technique so far. However, as palmtop computing becomes increasingly powerful and popular, the complexity of ANNs may become less onerous in real-time clinical settings. [4]

Furthermore, there are some theoretical advantages comparing a predictive ANN model over conventional models such as logistic regression.

One such advantage is that ANN model allows the inclusion of a large number of variables [28]. Another advantage of the neural network approach is that there are not many assumptions (such as normality) that need to be verified before the models can be constructed.

Although, one of the strengths of ANNs is their ability to still find patterns despite missing data, in this study a dataset with no related missing values was used.

Recently the task of comparison between these two models has been addressed from different points of view. Considering the publication bias, several published works in the medical literature have demonstrated the success of the ANN approaches. In a review carried out by Sargent on 28 major studies, ANN outperformed regression in 10 cases $(36 \%)$, was outperformed by regression in 4 cases $(14 \%)$ and the 2 methods had similar performance in the remaining cases. Sargent concluded that both methods should continue to be used and explored in a complementary manner. [29]

Gaudart et al. using simulated data, have compared the performance of ANN and linear regression models for epidemiological data and concluded that both had comparable performance and robustness and despite the flexibility of connectionist models (like ANN), their predictions were stable. [30]

This study was primarily designed to compare the performance of an ANN and a multivariable logistic regression analysis with the goal of developing a model for predicting the outcome in head injury and for studying their internal validity (reproducibility). Also setting up a standalone practical model for prediction of mortality in the head trauma patients was a secondary goal of this study.

Using freely downloadable software (PDP++) and making the networks and scripts accessible by the researchers can be perceived as an advantage of this study.

This study showed that ANN models significantly outperformed logistic models in both senses of discrimination and calibration, although lagged behind in accuracy. It is pointed out that the calibration values should be treated 
with some caution in this study, since according to Hosmer and Lemeshow, [31] in describing the statistic, HL statistic should be used where at least one of the predictor variables is continuous.

This study clearly shows that in a single comparison of these models based on the same data there is $22.2 \%$ chance of getting discrimination results contrary to our findings in majority of comparisons. This ratio is $43.6 \%$ for calibration and $32 \%$ for the accuracy results. These figures are practically important and imply that any single comparison between these two models cannot reliably represent their final performance.

Although considerable efforts, through many trial-anderrors, were made to optimize the design of the network, the designed ANN models could, and should, be further improved. In line with any other predictive models, likewise the findings of this study need to be externally validated. The networks are downloadable and the results can be studied in other study populations with divergent data and different survival ratios.

So far, there is no single algorithm that performs better than all other algorithms for any set of given head injury data. To this end, there is room for much more work to be done before a definite conclusion can be reached.

\section{Potential clinical use}

Should the results be reproducible in other populations, using a simple preprogrammed calculator (or other programmable computing devices) and minimal training of the personnel, this model and similar ones may emerge to be of considerable practical value in triage of the patients. At that time a dedicated instead of general purpose ANN software should be designed for this purpose.

The authors concur with the conclusion arrived by Tu [25] that logistic regression remains the clear choice when the primary goal of model development is to examine possible causal relationships among variables. However, it appears that ANNs or some form of hybrid technique incorporating the best features of both logistic regression and neural network models might lead to development of optimum prediction models for head injured patients.

\section{Conclusions}

In conclusion, this study compared models for the prediction of outcome in head injury using trauma data from hospital registries in Tehran, the data was applied to artificial neural network and multivariable logistic regression analysis. The predictive ability of the artificial neural network model was found to be comparable to that of the logistic regression model. Specifically, the ANN models significantly outperformed logistic models in both senses of discrimination and calibration but lagged behind in accuracy. Although the performance of the models were studied when the models were applied to the different samples of the original population study, external validation is necessary to get an accurate measure of performance outside the development population. Studies using larger databases with different rates of outcomes may further clarify the differences between artificial neural network and logistic regression models in head injury outcome prediction and their clinical implications.

\section{Competing interests}

The author(s) declare that they have no competing interests.

\section{Authors' contributions}

$\mathrm{BE}$ carried out the data extraction, performed the analysis and drafted the manuscript. KM supervised the analysis and critically reviewed the statistical viewpoints. HEA, MG and EK supervised the study and participated in its coordination. All authors read and approved the final manuscript.

\section{Acknowledgment}

The authors would like to express their gratitude to Mr. Ahmad Reza Eftekhar for his comments and assistance with the editing of this paper. We would also like to thank Mr. Sam Holden Research Assistant at the University of Sydney for his editorial help.

\section{References}

I. DiRusso SM, Sullivan T, Holly C, Cuff SN, Savino J: An Artificial Neural Network as a Model for Prediction of Survival in Trauma Patients: Validation for a Regional Trauma Area. J Trauma 2000, 49:2I 2-223.

2. Wyatt JC, Altman DG: Prognostic models: clinically useful or quickly forgotten? Brit M J 1995, 3 I I: I539- I54 I. (comment)

3. Greenwood D: An overview of neural networks. Behav Sci I99।, 36: 1 -33.

4. Terrin N, Schmid CH, Griffith JL, D'Agostino RB, Selker HP: External validity of predictive models: A comparison of logistic regression, classification trees, and neural networks. Journal of Clinical Epidemiology 2003, 56:721-729.

5. Patterson DW: Artificial Neural Networks: Theory and Applications. Englewood Cliffs, Prentice Hall 1996.

6. Leondes CT: Neural network systems, techniques, and applications. San Diego, Academic Press; 1998.

7. Baxt WG: Use of an artificial neural network for data analysis in clinical decision-making: the diagnosis of acute coronary occlusion. Neural Comput 1990, 2:480-9.

8. Baxt WG: Use of an artificial neural network for the diagnosis of myocardial infarction. Ann Intern Med 1990, I I 5:843-8.

9. Kemeny V, Droste DW, Hermes S, Nabavi DG, Schulte-Altedo-rneburg G, Siebler M, Ringelstein EB: Automatic embolus detection by a neural network. Stroke 1999, 30:807-10.

10. Das A, Ben-Menachem T, Cooper GS, Chak A, Sivak MV Jr, Gonet JA, Wong RC: Prediction of outcome in acute lower-gastrointestinal hemorrhage based on an artificial neural network: internal and external validation of a predictive model. Lancet 2003, 362(9392): | 26|-6.

II. Vijaya G, Kumar V, Verma HK: ANN-based QRS-complex analysis of ECG. J Med Eng Technol 1998, 22:160-7.

12. Kloppel B: Application of neural networks for EEG analysis. Consideration and first results. Neuropsychobiology 1994, 29:39-46. 
13. Jando G, Siegel RM, Horvath Z, Buzsaki G: Pattern recognition of the electroencephalogram by artificial neural networks. Electroencephalogr Clin Neuropsychol 1993, 86:100-9.

14. Penedo MG, Carreira MJ, Mosquera A, Cabello D: Computer-aided diagnosis: a neural-network-based approach to lung nodule detection. IEEE Trans Med Imaging 1998, 17:872-80.

15. Izenberg SD, Williams MD, Luterman A: Prediction of trauma mortality using a neural network. Am Surg 1997, 63:275-8I.

16. Li YC, Liu L, Chiu WT, Jian WS: Neural network modeling for surgical decisions on traumatic brain injury patients. Int J Med $\operatorname{lnf} 2000,57(1): 1-9$.

17. Grigsby J, Kooken R, Hershberger J: Simulated neural networks to predict outcomes, costs and length of stay among orthopedic rehabilitation patients. Arch Phys Med Rehabil 1994, 75: $1077-81$.

18. Tu JV, Guerriere MR: Use of a neural network as a predictive instrument for length of stay in the intensive care unit following cardiac surgery. Comput Biomed Res 1993, 26:220-9.

19. Nguyen T, Malley R, Inkelis S, Kuppermann N: Comparison of prediction models for adverse outcome in pediatric meningococcal disease using artificial neural network and logistic regression analyses. Journal of Clinical Epidemiology 2002, 55:687-695.

20. Dorsey SG, Waltz CF, Brosch L, Connerney I, Schweitzer EJ, Barlett ST: A neural network model for predicting pancreas transplant graft outcome. Diabetes Care 1997, 20: I 128-33.

21. Lang EW, Pitts LH, Damron SL, Rutledge R: Outcome after severe head injury: an analysis of prediction based upon comparison of neural network versus logistic regression analysis. Neurol Res 1997, 19:274-80.

22. Zargar M, Modaghegh MHS, Rezaishiraz H: Urban Injuries in Tehran: Demography of trauma patients and evaluation of trauma care. Injury 200I, 32(8):6I3-7.

23. Moini M, Rezaishiraz H, Zafarghandi MR: Characteristics and outcome of injured patients treated in urban trauma centers in Iran. J Trauma 2000, 48(3):503-7.

24. Des Plaines IL: Association for the Advancement of Automotive Medicine. Abbreviated Injury Scale: 1990 Revision. Association for the Advancement of Automotive Medicine 1990.

25. Tu JV: Advantages and disadvantages of using artificial neural networks versus logistic regression for predicting medical outcomes. I Clin Epidemiol 1996, 49: I225-3I.

26. Baxt WG: Application of artificial neural networks to clinical medicine. Lancet 1995, 346: I I35-8.

27. Ohno-Machado L, Rowland T: Neural network applications in physical medicine and rehabilitation. Am J Phys Med Rehab 1999, 78:392-8.

28. Bishop C: Neural networks for pattern recognition. Oxford University Press; 1995.

29. Sargent DJ: Comparison of artificial neural networks with other statistical approaches. Cancer (Supplement) 200I, 9I(8): 1636-42.

30. Gaudart J, et al.: Comparison of the performance of multi-layer perceptron and linear regression for epidemiological data. Computational Statistics and Data Analysis 2004, 44(4):547-570.

31. Hosmer D, Lemeshow S: Applied logistic regression. New York, Wiley; 2000

\section{Pre-publication history}

The pre-publication history for this paper can be accessed here:

http://www.biomedcentral.com/1472-6947/5/3/prepub
Publish with Bio Med Central and every scientist can read your work free of charge

"BioMed Central will be the most significant development for disseminating the results of biomedical research in our lifetime. "

Sir Paul Nurse, Cancer Research UK

Your research papers will be:

- available free of charge to the entire biomedical community

- peer reviewed and published immediately upon acceptance

- cited in PubMed and archived on PubMed Central

- yours - you keep the copyright

Submit your manuscript here:

http://www.biomedcentral.com/info/publishing_adv.asp
BioMedcentral 